\title{
Agôn
}

Revue des arts de la scène

5 | 2012

L'entrée en scène

\section{Enzo Cormann : Expériences poélitiques de l'entrée en scène}

\author{
Mireille Losco-Lena
}

\section{(2) OpenEdition}

Journals

Édition électronique

URL : http://journals.openedition.org/agon/2312

DOI : 10.4000/agon.2312

ISSN : 1961-8581

\section{Éditeur}

Association Agôn

\section{Référence électronique}

Mireille Losco-Lena, «Enzo Cormann : Expériences poélitiques de l'entrée en scène », Agôn [En ligne], 5 | 2012, mis en ligne le 25 janvier 2013, consulté le 24 avril 2019. URL : http:// journals.openedition.org/agon/2312; DOI : 10.4000/agon.2312

Ce document a été généré automatiquement le 24 avril 2019.

Association Agôn et les auteurs des articles 


\title{
Enzo Cormann : Expériences poélitiques de l'entrée en scène
}

\author{
Mireille Losco-Lena
}

1 La question de l'entrée en scène est récurrente, voire insistante, dans l'œuvre d'Enzo Cormann: elle constitue un motif que son théâtre, sa pensée sur le théâtre mais également ses romans se plaisent à convoquer et à interroger, le présentant sous des jours variés, le plaçant dans des configurations contradictoires et le déployant au sein d'un vaste champ d'expériences aussi bien fictionnelles que théoriques. Ce déploiement du motif fait de l'entrée en scène bien plus qu'une simple question de technique dramaturgique ou scénique, bien plus même qu'une question d'esthétique théâtrale. En reprenant un néologisme forgé par l'auteur, on peut dire que la question posée par ce motif est de nature "poélitique ", c'est-à-dire qu'elle engage l'ensemble du projet aussi bien poétique que politique du théâtre : poétique en ce que l'entrée en scène est l'amorce de toute fiction dramatique avec le surgissement du personnage sur le plateau, politique en ce que la bipartition entre scène et salle qu'elle génère suppose l'invention d'une relation de l'artiste au groupe et définit une configuration de pouvoir ${ }^{1}$. "Se demander d'où vient l'acteur $»^{2}$ est donc, pour Enzo Cormann, non seulement la première question du théâtre, mais sa question fondatrice, celle qui l'oriente tout entier et le rend possible - et parfois, comme nous aurons l'occasion de le constater, impossible ou intolérable. L'œuvre interroge alors moins l'apparition de ses personnages en tant que modalité esthétique de l'incipit théâtral que, plus profondément et plus viscéralement, la conception même du théâtre qu'une société se donne à elle-même. L'entrée en scène est en somme un révélateur du dispositif théâtral, envisagé dans ses dimensions à la fois dramaturgique et politique, et dont l'œuvre fait varier les possibles.

\section{Il vient de la salle}

Dans ses textes théoriques sur le théâtre, Enzo Cormann a proposé une modélisation théorique très claire de l'entrée en scène. En 2001, lors d'une conférence donnée à 
l'Université de Mexico, il explique que cette modélisation a d'abord surgi d'une suite de rêves à caractère archétypal :

Le rêve est sempiternellement le même : une foule est assise, muette. On pourrait croire que les gens prient, mais en réalité ils attendent seulement que quelque chose se passe. Quand je me lève au milieu d'eux, personne ne me remarque. Je les regarde, l'attente se mue en impatience. Quand je commence à leur parler, ils ne paraissent pas m'apercevoir. Leurs lèvres forment un à un les mots que je prononce. Quelque chose se dit par ma bouche, dont je ne distingue pas le sens. Quand je me rassois, les gens commencent à se parler. ${ }^{3}$

3 Un peu plus loin, Enzo Cormann livre une version plus inquiétante du rêve - "Je me réveille dans un hoquet de terreur ", conclut-il ${ }^{4}$ - dans laquelle l'attente de la foule fait pression sur lui : « les gens trépignent et sifflent $»^{5}$, lui intimant de prendre la parole. Il obéit et ne se contente plus de se lever «au milieu d'eux » pour parler: il «gagne la scène " puis il se " tourne vers la salle " ${ }^{6}$. Dès lors est déclenchée la possibilité de la fiction dramatique, qui ne sera plus seulement récit, mais également mise en jeu d'une fable, le récitant endossant le personnage.

4 Ces rêves archétypaux posent clairement les données théoriques de l'entrée en scène telle que la conçoit l'auteur. Premier point: l'«artisan $»^{7}$ de théâtre - auteur et acteur superposés par le rêve en une seule fonction archaïque - se situe au milieu d'un groupe auquel il appartient, et qu'Enzo Cormann baptise « l'assemblée théâtrale ». Il ne vient pas d'ailleurs: la coulisse d'où il peut techniquement surgir n'est pas un autre monde coupé de celui-ci et nimbé de mystère ${ }^{8}$ : elle appartient à l'enceinte de cette assemblée-ci. En somme, sortir de la coulisse équivaut toujours à venir de la salle : l'entrée en scène ne saurait être pensée sur le modèle de l'épiphanie. Deuxième point : le désir de l'entrée en scène, en tant que commencement du théâtre, n'appartient pas (seulement) à celui qui se lève pour monter sur la scène : il correspond à une attente collective et cette entrée est même « requis $[\mathrm{e}] »^{9}$ par l'assemblée. Troisième point : si « personne ne me remarque » et si «quelque chose se dit par ma bouche », c'est que l'artisan de théatre n'entre pas en scène pour se faire valoir, mais, comme l'énonce clairement une série de formules familière à Enzo Cormann, pour parler "pour le compte» de l'assemblée, pour être le "délégué » de l'assemblée ${ }^{10}$. Sortir de la foule n'est pas s'en extraire pour s'élever sur les divines planches du théâtre, mais être "provisoirement délégué » par l'assemblée pour raconter et jouer des fictions qui la concernent.

5 Cette conception de l'entrée en scène - et partant, du projet même du théâtre - s'inscrit clairement dans une période de l'histoire de la scène occidentale ouverte à la fin du XIX siècle, avec les premières virulentes contestations contre le cabotinage des acteurs. Elle relève de la tradition, toujours fragile, du théâtre public et populaire, opposée à la conception à la fois aristocratique et bourgeoise de l'entrée en scène de l'acteur comme " apparition », liée à ce que Zola nommait avec ironie «la pompe de la représentation » ${ }^{11}$. Une viscérale horreur de cette théâtralité-là habite la pensée théâtrale d'Enzo Cormann, qui a souvent recours à la métaphore animalière pour l'évoquer. Ainsi du caniche dans le Dit de l'impétrance, où il est question de l'acteur de cinéma se rêvant en vedette :

regardez-moi ! admirez-moi ! jappe le caniche dressé sur ses pattes arrières / tour de piste et susucre / prix Machin ou médaille ou fric / ma gueule dans les journaux et mon nom sur l'affiche ${ }^{12}$

6 À l'opposé, l'acteur délégué par l'assemblée sera défini comme un "passeur", un "conducteur » ${ }^{13}$ caractérisé par l'«abnégation», le «renoncement joyeux à la confiscation individuelle de l'œuvre au profit d'une co-opération du singulier et du 
pluriel $»^{14}$. On a ici l'élaboration d'une forme de contrat social, fondateur d'une pratique artistique d'essence démocratique.

\section{Comparution pour interrogatoire}

7 Mais de quel pouvoir l'acteur entrant en scène est-il le délégué démocratique ? Enzo Cormann le définit à travers une série d'images volontiers scientifiques, qui apparentent l'acte théâtral à un acte clinique : l'assemblée démocratique, telle que la conçoit en effet la pensée politique occidentale moderne, exige l'exercice d'une pensée éclairée et elle s'outille des lumières d'une raison analytique. Ainsi l'entrée en scène cormannienne initie-t-elle un processus d' " examen » conçu comme une " opération », comparable à une «leçon d'anatomie $»^{15}$; elle est également "expérimentation $»^{16}$, organisation d'une expérience sur les affaires humaines. L'entrée en scène de l'acteur, en déclenchant la fiction dramatique, est une manière de coup de scalpel amorçant la dissection d'un corps - celui du personnage et de son histoire - pour en prendre connaissance, ou du moins en interroger les énigmes, tout cela pour le compte de l'assemblée.

Ce geste est très sensible dans les pièces de l'auteur. Diktat (1995), par exemple, est une partition théâtrale pour deux acteurs mettant en scène deux demi-frères qu'une guerre nationaliste aux accents balkaniques a séparés, et qui reviennent depuis leur mort pour analyser devant nous et pour nous le corps tragique de leur conflit. Ces deux êtres de fiction, Piet et Val, sont appelés à entrer en scène alors qu'ils sont supposés être déjà morts, et la pièce que forme le déploiement de leurs paroles est une autopsie en mouvement guidée par une "simple» question ainsi formulée: "Qu'est-ce qui se passe ? $\aleph^{17}$. Ce mode interrogatif, sur lequel se joue fondamentalement l'entrée en scène cormannienne, inscrit celle-ci dans un paradigme non seulement politique, mais également cognitif: le drame est une pensée au travail et non pas ce que Peter Szondi nommait un «drame absolu », le développement d'une action au présent ${ }^{18}$. Ce théâtre de la pensée, ou mieux: ce théâtre de la question, toujours prêt au débat contradictoire autre motif essentiel à l'exercice démocratique du pouvoir -, entrelacera le présent et le passé sans chercher à justifier de façon réaliste ces jeux de distorsions et de jonctions temporelles. L'endroit de la question qu'est la scène théâtrale s'autorise en effet toutes les « comparutions $»^{19}$ : comparution, par l'intermédiaire des acteurs, de deux personnages tantôt morts et se racontant au présent («je m’adresse à vous dans le temps figé de ma propre mort " déclare Piet dans la première tirade de la pièce ${ }^{20}$ ), tantôt vivants et dialoguant dans un passé rejoué pour nous. L'important n'étant pas de faire illusion, mais de déployer de l'espace-temps expérimental.

9 L'expérimentation, selon Claude Bernard, l'un de ses grands théoriciens, est un travail de "reconstitution" qui ne saurait être confondu avec une simple réitération. La reconstitution expérimentale d'un événement est en effet une façon de le re-jouer en l'observant et en l'interrogeant : l'expérimentation, insiste Claude Bernard, est guidée par une idée, ce qu'il nomme une "hypothèse $»^{21}$. Le bon expérimentateur est celui qui sait poser les bonnes questions devant les phénomènes. Les jeux temporels de Diktat sont de ce point de vue des reconstitutions expérimentales: le passé représenté relève moins stricto sensu d'un phénomène de flash-back que d'une "opération" de reconstitution, conduite sur le mode interrogatif. Le passé n'est ni évoqué, ni remémoré, ni revécu : il est appelé à comparaître pour interrogation. Dans ce que Jean-Pierre Sarrazac nomme une « rétrospection $»^{22}$, le passé scéniquement reconstitué n'est pas la redite du passé 
supposément vécu par les deux personnages morts ; il est le passé vécu plus la pensée qui le dissèque à l'aide de bonnes questions. Cette réalité augmentée par la question, Enzo Cormann la baptise "l'explication $»^{23}$. "Nous attendons du théâtre, écrit-il ainsi, qu'il revienne sur l'expérience $»^{24}$. Dans une pièce plus récente, L'Autre, sous-titrée justement "Trois explications» (2006), deux femmes dialoguent pour disséquer leur vie en en défaisant les plis, jusqu'à parvenir au « moment » de « poser la question $»^{25}$; l'explication apparait alors moins comme un enseignement didactique que comme un processus d'enquête.

En faisant comparaître le passé, l'expérimentation dilate les événements et elle se donne de l'espace, du temps mais aussi de l'intelligence - cette raison éclairée sans laquelle il n'est pas de pouvoir démocratique possible. L'expérimentateur doit donc donner : non pas se donner soi-même tout entier dans le jeu théâtral, comme certains acteurs aiment à le penser, mais se doter de cette lumière prométhéenne permettant d'instituer l'examen théâtral. Les personnages cormanniens acquièrent ainsi souvent des statures titanesques par l'intelligence des mots qui leur sont prêtés, par la puissance et la générosité de leur réflexion, de leur culture, de leur clairvoyance, et également par la force de leurs émotions. Ils ne sont pourtant pas des héros au sens traditionnel du terme, c'est-à-dire des êtres supérieurs renvoyant à une conception inégalitaire du pouvoir ; ils sont plutôt animés du feu de l'expérimentation dont ils se trouvent être les supports, pour le compte de l'assemblée théâtrale. Le personnage de Cairn, dans la pièce éponyme (1997), est à la fois, au niveau de la fable, un syndicaliste mobilisé contre la fermeture de son entreprise pour cause de délocalisation, et en même temps, au niveau de l'examen théâtral, une figure engagée dans un combat de Titans, animé d'une puissance de parole plutôt hors norme dont la fonction, non illusionniste, est d'éclairer la situation dramatique. En dotant les personnages du feu prométhéen - cette prolixité vertigineuse, à la fois jouissive et inquiète, qui fait le style d'Enzo Cormann -, la dramaturgie en fait ainsi les sujets d'une dissection conduite sur eux-mêmes.

\section{Hoquet de terreur}

11 La puissance de cette lumière prométhéenne, pensée comme un bien commun au sein de l'assemblée théâtrale, n'empêche pourtant pas que l'entrée en scène puisse parfois s'accompagner d'un « hoquet de terreur». Terreur nocturne qui ourle l'examen clinique, s'inscrit dans certains de ses plis, ébranle le sujet appelé à comparaître sur la scène. Il y a en effet une menace en latence dans l'entrée en scène cormannienne. Si la deuxième version du rêve raconté dans A quoi sert le théâtre? nous en prévenait ( Je me réveille dans un hoquet de terreur »), l'œuvre dramatique et romanesque en déploie plusieurs versions : l'entrée en scène, alors thématisée par la fable et faisant elle-même l'objet du drame, apparaît dans sa dimension de cauchemar, et ce cauchemar se joue à l'endroit d'une terreur politique.

«Theo Steiner joua quatre fois Macbeth au Burgtheater de Vienne, et ne se présenta pas à la cinquième, ni à aucune des suivantes $[. ..] \aleph^{26}$. Ainsi s'énonce, dans Toujours l'orage (1997), l'énigme de l'impossible cinquième entrée en scène d'un grand acteur juif d'après-guerre, Theo Steiner. Cette pièce est un long combat dialogué entre lui et un metteur en scène de la génération suivante, venu le dénicher dans la campagne française où il se terre depuis une vingtaine d'années, pour tenter de le persuader de remonter sur les planches et de jouer le personnage de Lear. Ce projet n'aboutira pas, mais il nous offrira matière à 
l'examen de cette impossible cinquième entrée en scène - et de l'horreur dont elle s'avère le symptôme. Ce qui n'a pu se mettre en mots, au moment de cette "non entrée en scène ", la pièce tâche alors de le "déplier $~^{27}$ dans un long dialogue analytique qui remontera jusqu'au camp de Terezin pendant la Seconde Guerre mondiale, camp où les nazis encouragèrent les artistes à pratiquer leur art, musique, opérettes, théâtre. Le cauchemar de Theo Steiner, sauvant sa peau en se compromettant avec le nazisme, amorçant précisément sa carrière d'acteur à partir de cette compromission, s'inscrit dans "l'aire indéfinie de l'ambiguïté et du compromis» nommée par Primo Levi la "zone grise $\aleph^{28}$. Dans ce contexte, entrer en scène fonctionne comme un véritable symptôme de l'histoire où se désigne un indicible : le "ça " évoqué par Karl Krauss dans Les Derniers jours de l'humanité et placé en exergue de Toujours l'orage : «Ce n'est pas que vous ayez dû mourir, mais que vous ayez dû vivre ça, voilà qui, à l'avenir, fera que tout sommeil, toute mort dans un lit douillet, aura l'air d'un péché »"29. Incapable de penser ce «ça ", Theo Steiner n'aura pu que substituer à son entrée en scène, ce cinquième soir-là, une longue fuite aux accents du troisième acte du Roi Lear, où le vieux roi fou erre dans la lande tandis que sévit « toujours l'orage».

13 Et si Terezin se poursuivait toujours potentiellement, par delà l'Histoire, dans chaque salle de théâtre? Autrement dit, pour le compte de quelle assemblée l'acteur entre-t-il en scène, et quel contrat politique lie le singulier au collectif? «Je vis le public, figurez-vous, le public à l'image du peuple, le peuple à l'image du bourreau $\|^{30}$, explique Theo Steiner à Nathan Goldring. Toujours l'orage parle de la haine d'un théâtre inextricablement lié au pouvoir et complice malgré lui des bourreaux. "De quelle nouvelle barbarie serai-je le bouffon? $\|^{31}$ se (nous) demande ainsi Goldring au terme de la pièce : cette question met des mots sur le hoquet du cauchemar qui accompagne toute entrée en scène, en tant qu'elle est potentielle réitération d'une compromission dévastatrice.

Par son dispositif analytique, Toujours l'orage n'est pas seulement une pièce de théâtre sur le théâtre ; c'est également le déploiement d'un conflit entre la thématique de la pièce (impossible entrée en scène, horreur du théâtre) et son projet (faire malgré tout théâtre de cet impossible, entrer malgré tout en scène). Ce que la perversité nazie a fait de Steiner, la pièce de Cormann travaille à le dénouer. Non pas psychologiquement, car il ne s'agit pas d'un processus qui viserait à apaiser les tourments de Steiner, mais " poélitiquement »: le théâtre en tant qu'examen met de la lumière dans les plus sombres questions de l'histoire; Steiner est moins une personne réelle qu'il n'est l'« outil » d'une analyse lumineuse qui ne renonce pas à la puissance de la question. Le personnage est alors "réenvisagé comme sujet du drame " ${ }^{32}$, arraché, grâce à l'espace-temps expérimental de la scène, à la zone grise où il s'est perdu en tant qu'acteur. En faisant comparaître l'impossible entrée en scène de Steiner, Toujours l'orage s'affirme ainsi comme un métadrame: la pièce invente la scène secondaire de l'examen démocratique pour faire comparaître la scène primaire d'un effroyable symptôme politique. Et ce métadrame peut alors être conçu comme un lieu de déprise de l'aliénation.

Le conflit profond entre sujet et sujétion, qui organise la dramaturgie de Toujours l'orage, met en tension deux conceptions antagonistes et irréconciliables de l'entrée en scène: d'un côté, celle-ci est envisagée comme une hypothèse lumineuse que l'assemblée théâtrale se donne à elle-même ; de l'autre, elle est une aliénation mortifère engageant un rapport de pouvoir entre scène et salle, artiste et assistance. On pourrait dire encore, en suivant une autre opposition faite par Enzo Cormann, que la première entrée en scène est l'entrée en scène théâtrale par excellence, tandis que la seconde est l'entrée en scène de 
spectacle $^{33}$ : au sujet théâtral de l'opération s'oppose un objet spectaculaire soumis au regard aliénant, réifiant, du public. Cette opposition est au centre de La Révolte des anges (2004), qui met en scène la révolte de trois figures d'artistes défunts (Koltès, Basquiat et Baker) contre l'idolâtrie d'un public qui les a figés en icônes. Ils disent l'horreur de ce que le succès a fait d'eux des "êtres pour-autrui ", comme disait Sartre : des objets du regard de l'autre, objets de spectacle donc, réifiés de ce regard faussement admiratif et vraiment mortifère. Entrer en scène, c'est alors appartenir au regard de l'autre - du fan, de la groupie, déjà tout prêts à applaudir (mais, comme l'indique l'adage russe placé en exergue de Cairn, "prends garde que ta tête ne se trouve entre les mains de ceux qui t'applaudissent $\aleph^{34}$ ). Entrer en scène, pour le dire autrement, c'est ni plus ni moins être "fourrés dans le cul de l'espèce comme une boulette d'opium " ${ }^{35}$. Cette Révolte des anges, qui fait entendre un cri d'outre-tombe contre la puissance d'un monde marchand, fait théâtre d'une révolte contre le "devenir-machin» du théâtre ${ }^{36}$. Elle poursuit le conflit, déjà exploré dans Toujours l'orage, entre le thème (aliénation de l'art et/ou de l'artiste) et le projet de la pièce (tentative de contrer, de combattre l'aliénation); mais à la configuration politique de Toujours l'orage succède une configuration socio-économique, caractérisée par ce que l'auteur appelle une « réification générale des rapports sociaux de production artistique $\aleph^{37}$. On connaît la célèbre critique brechtienne de la théâtralité bourgeoise aliénant le public ; Enzo Cormann y adjoint une critique du théâtre aliénant celui qui se risque sur les planches: "On pourra bien dire l'acteur "merveilleux" ou "bouleversant", il n'en sera pas moins le jouet d'une foule, elle-même jouet des marchands. Et plus l'acteur en joue, plus il est joué. Plus il en fait, plus il est fait. $~^{38}$

\section{Échapper (belle)}

Dans son dernier roman, Vita Nova jazz (2011), Enzo Cormann revient encore une fois sur le motif de la (non) entrée en scène. Après dix ans de silence, le saxophoniste Jim Erris a accepté de reformer son groupe, Vita Nova jazz ensemble, pour un unique concert dans un grand festival. Mais au moment de son entrée en scène, alors que la salle est remplie à craquer et que l'organisateur du festival piaffe, presse, puis supplie Jim, le musicien plante tout le monde et s'enfuit. Cette impulsion violente, irrésistible, à ne pas entrer en scène, fait à nouveau symptôme : Jim Erris qui, dix ans plus tôt, a failli tuer sa femme dans un accès de fureur, est dans l'impossibilité de reprendre le fil de sa vie, de faire comme si rien ne s'était passé. Le roman déploie alors la violente contradiction, dont la non-entrée en scène est le symptôme, entre la logique intime et singulière de l'artiste, en quête d'une " vie nouvelle ", et la logique commerciale des impresarios, celle du come back prestigieux et juteux - d'un grand nom du jazz. Le portrait ironique et dégoûté que Jim Erris dresse du producteur du festival, Massimiliano Forlano, est sans équivoque : s'y dit la détestation viscérale du «marchand » et de la situation de l'art dans les mains des marchands. « Dans la vraie vie toute ma peinture crache sur les marchands / Et dans la véritable vie toute ma peinture est aux mains des marchands ", dénonçait déjà l'Enfant radiant (Jean-Michel Basquiat) de La Révolte des anges ${ }^{39}$. Le seul pouvoir de l'artiste, contre la logique commerciale du spectacle, serait-il donc de déguerpir au moment d'entrer en scène?

17 À la différence de Toujours l'orage, Vita Nova jazz ne propose pas l'analyse métadramatique du refus de l'entrée en scène. Jim Erris quitte la scène pour écrire, dans la solitude, une longue lettre à son ancienne femme et c'est ce texte qui constitue le roman. Comme dans les deux autres volets de la trilogie, l'écriture romanesque est ainsi pensée comme 
solitaire et adressée à un destinataire précis. Elle constitue en cela un geste hétérogène au geste théâtral : le roman est le lieu d'une écriture qui n'a pas besoin de l'assemblée. Certes, le romancier souhaite certainement être lu par des lecteurs, mais l'existence de ses romans n'est pas conditionnée à l'existence d'un lectorat. En revanche, aime à dire Enzo Cormann, il est inconcevable d'écrire $d u$ théâtre: on ne peut qu'écrire pour le théâtre; en d'autres termes, le théâtre n'est pas sans l'assemblée. Avec ses trois romans à ce jour publiés, Le Testament de Vénus (2006), Surfaces sensibles (2007) et Vita Nova jazz, l'auteur ouvre ainsi un espace d'écriture où, justement, se racontent des histoires d'artistes solitaires, libres du regard - et de l'argent - du public. Vénus, dans Le Testament de Vénus, est un poète et artiste d'art brut qui non seulement refuse de vendre ses œuvres, mais décide également de les brûler avant de mourir. De la même façon, dans Surfaces sensibles, le personnage de Zoé est une musicienne qui ne joue qu'avec casque et qui, dans sa claustration psychotique, ne donne à entendre ses compositions à personne. Mais cette solitude n'empêche pas les artistes de faire œuvre.

Le passage d'Enzo Cormann à l'écriture romanesque, campant des artistes coupés du commerce - au sens étroit comme au sens le plus large du terme - avec leurs contemporains, n'est certainement pas insignifiant. Sa trilogie romanesque donne voix à une impulsion proprement contradictoire au théâtre, au fait théâtral lui-même. Cette impulsion, déjà thématisée dans Toujours l'orage, substitue au motif de l'entrée en scène celui de l'échappée belle, expression récurrente sous la plume de l'auteur : « Ma mission sur cette Terre : être et n'être pas, échappée belle ! OUI ET NON, O FOOL! I SHALL GO MAD! Lear à Terezin, Lear à Berlin, Never, never, never, never, never! $»^{40}$ Si la « ROUTE » du théâtre « EST PAVÉE DE FOLIE », alors «ÉVITONS-LÀ », déclare encore Theo Steiner citant Shakespeare ${ }^{41}$. L'impulsion de l'échappée belle joue dans l'œuvre en opposition avec le mouvement de la comparution et dote l'écriture d'une autre énergie : celle de la folie, non domestiquée par quelque dispositif que ce soit, l'énergie de la révolte, de la violente pulsion à se rebiffer - même si, comme dans Cairn, c'est la mort qui est au bout de l'échappée belle. Ne pas/plus entrer en scène, alors, c'est faire exploser le dispositif raisonné du théâtre et y amener cette part de sauvagerie sans laquelle, peut-être, il risque à tout moment de se faire l'« auxiliaire des chiens de garde $»^{42}$.

Cette dimension sauvage, évidente chez les figures d'artistes de la trilogie romanesque, s'inscrit en décalage avec l'imaginaire clinique de l'œuvre et donne à entendre la part de l'imaginaire américain qui la traverse. L'échappée belle, c'est le road movie, déraisonnable et viscéral, qui entremêle la jouissance de la vie avec le risque catastrophique de la mort (une séquence de La Révolte des anges, intitulée précisément "Road movie », lâche ainsi la bride à la dangereuse ivresse de vivre) ; c'est aussi la présence de Jack Kerouac en figure tutélaire, qui fait signe de façon récurrente dans l'œuvre ${ }^{43}$. Le dispositif de la comparution se voit alors contrarié, ou problématisé, par la tentation du vagabondage solitaire, pour paraphraser le titre du livre de Kerouac précisément posé sur le chevet de Cairn. La dramaturgie de Cairn est à ce titre tout à fait significative : les deux premières parties de la pièce, dans le droit fil de pièces comme Diktat ou Toujours l'orage, constituent une mise en examen (ici des ravages du libéralisme) par le combat dialogué des personnages. Mais succède à ce dispositif, dans la dernière partie de la pièce, l'échappée belle - et noire - du syndicaliste Cairn, écrasé par la machine économique et le cynisme tout puissant de ses adversaires. Cairn bifurque alors vers le vagabondage solitaire parce qu'il n'est plus temps de "s'expliquer » : à continuer son « commerce » avec les patrons, le syndicaliste risque de n'être plus que l'« auxiliaire des chiens de garde ». C'est bel et bien sous le signe 
de Kerouac que s'enclenche la fin de la pièce, road movie catastrophique où les vapeurs de l'alcool se mêlent au bruit des armes à feu, dérive en gestes incendiaires et tentations meurtrières qui s'achève, comme l'exige le genre, dans la mort. La mixité dramaturgique de la pièce met de toute évidence en crise le dispositif de la comparution pour le problématiser : elle semble poser la limite poélitique à partir de laquelle le théâtre se doit, éthiquement, politiquement ou tout simplement humainement, de donner voix à l'impérieuse impulsion de l'échappée.

\section{Points de fuite}

Le road movie relève du roman, de la poésie et du cinéma; il n'est pas un genre théâtral. C'est dans l'ailleurs du théâtre que le théâtre d'Enzo Cormann va ainsi puiser. Ce faisant, il affirme la nécessaire inscription, dans l'espace-temps de la scène, de points de fuite dramaturgiques. Cairn ne recourt plus au métadrame analysant de ce qu'entrer en scène (ou pas) veut dire; la pièce agence plutôt un jeu de forces contradictoires en vertu desquelles l'entrée en scène s'accompagne de la possibilité de s'en échapper, fût-ce dans le tragique. Par-delà Cairn, l'ensemble du théâtre d'Enzo Cormann se plait à explorer ce jeu contradictoire - et complémentaire - entre la saisie clinique et l'échappée lyrique, entre l'art de l'entrée en scène comme acte collectif et l'art de la fugue solitaire comme porte de sortie. Au fil des pièces, la dramaturgie cormannienne travaille au corps le modèle expérimental de la comparution en le dotant d'une multiplicité de points de fuite, en sorte que l'œil clinique ne puisse pas se mettre au service de la barbarie (politique, économique, spectaculaire), qu'il trouve toujours le moyen de s'échapper du côté du vagabondage ou de la révolte - même si c'est la nuit noire qu'il rencontre.

Il est possible que l'écriture en versets, qui a succédé aux denses proses de l'auteur dans ses pièces les plus récentes ${ }^{44}$ et qui ouvre dans l'écriture dramatique la fenêtre lyrique de ses jazz poems ${ }^{45}$, relève de cette puissance du point de fuite dans le dispositif théâtral: scandant la parole en unités de souffle, le verset entrelace la pause respiratoire aux mots, le silence à la parole. Quelque chose se tait ou échappe au dire de l'intérieur même de ce qui se dit. Mais plus significative encore, et surtout plus constante dans l'œuvre dramatique depuis ses débuts, est la part d'énigme et d'altérité dont les personnages sont tissés : dans les "concerts d'enfers" que sont les pièces sur Sade (Sade, concert d'enfer, 1989) et Gilles de Rais (La Plaie et le couteau et L'Apothéose secrète, 1993), et jusqu'aux pièces les plus récentes, L'Autre ou Bluff (2012), la comparution des personnages est problématisée par l'abyssale énigme de l'être intime. La dissection est troublée par le constat du monstrueux ou de l'inconnaissable, ce qui de l'être ne saurait être saisi ni par les autres, ni par lui-même :

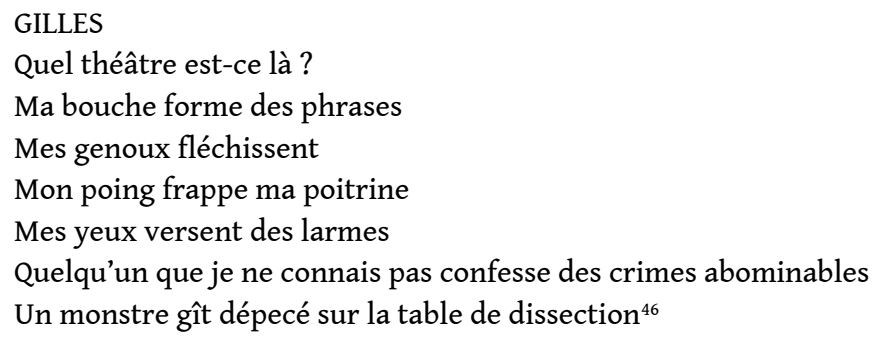

Le théâtre d'Enzo Cormann convoque sur la table de dissection la part du monstrueux qui pourtant ne se dissèque pas ; il expose à la lumière la part de l'ombre qui ne s'éclaire pas. Dans l'avertissement de La Plaie et le Couteau, l'auteur, reprenant Bataille, affirme ainsi que 
sa pièce "ne prétend nullement explorer la nuit: c'est la nuit qui l'explore $»^{47}$. Aux lumières prométhéennes de l'examen répond, comme en écho contradictoire, l'expérience de la nuit - cette nuit dont « l'énigme sans appel » du crime des sœurs Papin, qui a passionné l'auteur ces dernières années, constitue peut-être l'exemple paradigmatique ${ }^{48}$. Quelque chose échappe à cela même qui est appelé à comparaître ; quelque chose dont le silence, pourtant, "ne saurait être vide, même s'il était obscur ", pour reprendre la célèbre formule de Lacan à propos du crime des sœurs Papin ${ }^{49}$. La scène du crime, mais également la scène psychique des deux sœurs, sont précisément des scènes impénétrables, closes sur leur mystère. Dans ce qui, au final, semble relever d'une dramaturgie du clair-obscur - lumière de l'examen démocratique, nuit de la monstrueuse humanité -, l'entrée en scène cormannienne nous invite à faire l'expérience qu'il y a quelque chose que nous ne pouvons pas connaître ni nommer, qu'il y a du «manque-àpenser $»^{50}$ au cœur de toute comparution humaine.

L'œuvre d'Enzo Cormann peut ainsi se lire comme une longue expérience ou une suite d'expériences de ce qu'entrer en scène veut dire: l'auteur explore ce qui se joue dans l'instant où quelqu'un se lève du groupe pour gagner le plateau, se retourner et initier le jeu de la fiction dramatique. Sa pensée - qu'elle soit théorique ou fictionnelle - s'enrichit des configurations contradictoires qu'elle rencontre en déployant ainsi le champ des possibles, et pour ainsi dire en le cartographiant: ici un acteur monte sur les planches pour être applaudi par des nazis, là des artistes reviennent d'entre les morts pour protester contre leur réification, là encore un musicien s'enfuit juste au moment d'entrer en scène. Ailleurs, d'autres ignorent la scène et vivent leur trajectoire de vagabonds solitaires, payant parfois leur liberté du prix de la mort. Si l'entrée en scène cormannienne s'inscrit clairement dans un double paradigme politique et cognitif, l'œuvre en expérimente les contrastes, les complexités et les apories: la comparution éclairée, à la fois source de compréhension du point de vue cognitif et émancipatrice du point de vue politique, y affronte la nuit de l'aliénation et de l'inconnaissable. Le rêve fondateur du désir de théatre, porté chez Enzo Cormann par une profonde conviction démocratique, peut ainsi virer au cauchemar dès lors que les pouvoirs du théâtre se trouvent confisqués par les tyrans ou les marchands; l'examen théâtral peut tourner court lorsqu'il n'est plus temps de "s'expliquer » et qu'il faut songer à s'échapper (belle) ; la dissection éclairée bute sur l'énigme sans appel, et le feu prométhéen reconnaît la puissance de la nuit. Et pourtant, Enzo Cormann ne renonce jamais à l'horizon utopique de cette scène qui le poursuit partout, théâtre, romans, essais : en faisant de l'entrée en scène un véritable champ d'expériences, il met à l'épreuve son propre rêve théorique pour mieux en affirmer, semble-t-il, l'impérieuse nécessité - et pour mieux en réinventer, peut-être, les possibilités. 


\section{NOTES}

1. Sur l'adjectif «poélitique », voir Cormann, Enzo. Ce que seul le théâtre peut dire. Considérations poélitiques. Besançon: Les Solitaires intempestifs, 2012, notamment «Considérations poélitiques. Six notes sur la gageure théâtrale ", p. 33-60.

2. Id., p. 33.

3. Cormann, Enzo. "À quoi sert le théâtre? » In: À quoi sert le théâtre? Besançon: Les Solitaires intempestifs, 2003, p. 85.

4. Id., p. 88 .

5. Ibid.

6. Ibid.

7. Enzo Cormann revendique le terme d' " artisan de théâtre », id., p. 12.

8. Cormann, Enzo. «Considérations poélitiques. Six notes sur la gageure théâtrale ». In: Ce que seul le théâtre peut dire, op. cit., p. 33.

9. Id., p. 87.

10. Toutes ces expressions sont récurrentes dans les textes théoriques d'Enzo Cormann et parcourent les deux volumes publiés aux Solitaires intempestifs et déjà cités; on pourra tout particulièrement se reporter à « L'examen théâtral » et « À quoi sert le théâtre ? ». In: À quoi sert le théâtre?,op. cit.

11. Zola, Émile. Le Naturalisme au théâtre [1881]. Bruxelles: Complexe, 2003, p. 120. Je renvoie à la très belle page que consacre Enzo Cormann à cette théâtralité, dans «Considérations poélitiques ", op. cit., p. 34 .

12. Cormann, Enzo. Le dit de l'impétrance. Je m'appelle et autres textes. Paris: Minuit, 2008, p. 90.

13. Cormann, Enzo. "Cette incompréhensible distraction de soi d'avec soi. Huit notes sur l'abnégation de l'acteur ». In: Ce que seul le théâtre peut dire, op. cit., p. 159.

14. Cormann, Enzo. «Nous sommes tous des groupuscules! Sept notes sur un projet de fédération (de groupes et d'outils) de théâtre », id., p. 186.

15. Voir Cormann, Enzo. "L'examen théâtral» et «À quoi sert le théâtre? ». In: À quoi sert le théâtre?, op. cit.

16. Voir par exemple Cormann, Enzo. «L'opération théâtrale. Dix notes sur ce que seul le théâtre peut dire ». In: Ce que seul le théâtre peut dire, op. cit., p. 71.

17. Cormann, Enzo. Diktat, Paris: Minuit, 1995, p. 13 et p. 80.

18. Szondi, Peter. Théorie du drame moderne, traduit de l'allemand par Sybille Muller. Belval: Circé, coll. « Penser le théâtre », 2006. [1956]

19. Terme qu'Enzo Cormann emprunte à Jean-Luc Nancy; «Crise de la représentation/ représentation de la crise. Douze notes pour un théâtre de crise ». In: Ce que seul le théâtre peut dire , op. cit., p. 108.

20. Cormann, Enzo. Diktat, op. cit., p. 11.

21. Bernard, Claude. Introduction à l'étude de la médecine expérimentale. Paris: Le Livre de Poche. [1865]. P. 136.

22. Cormann, Enzo et Sarrazac, Jean-Pierre. «Le Mouvementeur. (Entretien)». In: Enzo Cormann. Le Mouvementeur, Registres Hors série II, Presses de la Sorbonne Nouvelle, printemps 2010. P. 106. Sur la dramaturgie rétrospective, voir également Sarrazac, Jean-Pierre. Théâtres intimes. Arles: Actes Sud, coll. « Le temps du théâtre », 1989.

23. Voir par exemple « L'opération théâtrale », op. cit., p. 64. 
24. Cormann, Enzo . «Crise de la représentation/représentation de la crise. Douze notes pour un théâtre de crise », op. cit., p. 92.

25. Cormann, Enzo. L'Autre. Trois explications, Paris: Minuit, 2006, p. 52.

26. Cormann, Enzo. Toujours l'orage, Paris: Minuit, 1997, p. 19.

27. Selon un autre terme cher à l'auteur. Voir par exemple son entretien avec Jean-Pierre Sarrazac, « Le mouvementeur (Entretien)», op. cit., p. 106-108.

28. Levi, Primo. Les Naufragés et les rescapés, Quarante ans après Auschwitz, Paris, Gallimard, coll. «Arcades », 2003. [1986]. P. 66.

29. Cormann, Enzo. Toujours l'orage, op. cit., p. 7.

30. Id., p. 87.

31. Id., p. 88 .

32. «Figures ou personnages perpétuellement réenvisagés comme sujets du drame, davantage qu' objets d'étude", revendique Enzo Cormann dans "Quelque chose d'autre. Cinq notes sur le modèle mort-vivant ». In: Ce que seul le théâtre peut dire, op. cit., p. 168.

33. Voir Cormann, Enzo. "Sous le regard des anges. Quatre notes sur la modernité théâtrale ». In: Ce que seul le théâtre peut dire, op. cit., p. 28.

34. Cormann, Enzo. Cairn. Paris: Minuit, 1997, p. 9.

35. Cormann, Enzo. La Révolte des anges. Paris: Minuit, 2004, p. 66.

36. Cormann, Enzo. «Considérations poélitiques », op. cit., p. 35.

37. Cormann, Enzo. « Sous le regard des anges », op. cit., p. 28.

38. Cormann, Enzo. «Considérations poélitiques », op. cit., p. 35.

39. Cormann, Enzo. La Révolte des anges, op. cit., p. 63.

40. Cormann, Enzo. Toujours l'orage, op. cit., p. 69.

41. Id., p. 23.

42. " Le théâtre comme parfait auxiliaire des chiens de garde ", selon la formule de Theo Steiner, id., p. 56.

43. Figure à laquelle est consacré Le Dit de la chute. Tombeau de Jack Kerouac. In: Enzo Cormann. Le mouvementeur, op. cit., p. 79-93 ; voir également Tombeau de Jack Kerouac, jazz poem d'Enzo Cormann (texte, voix), Jean-Marie Machado (piano) et Jean-Marc Padovani (saxophones), CD édité par Soléart productions/La Grande Ritournelle, 2009. Il n'est pas anodin que ce jazz poem évoque un Kerouac «entrant soûl chaque soir en scène» au Village Vanguard en 1957(Enzo Cormann. Le Mouvementeur, op. cit., p. 79), et que ces entrées en scène catastrophiques soient liés au motif de sa chute.

44. Exception faite de L'Apothéose secrète, "poème dramatique" écrit en vers libres et courts (Cormann, Enzo. La Plaie et le Couteau, suivi de L'Apothéose secrète. Paris: Minuit, 1993).

45. Forme poétique et musicale pratiquée par Enzo Cormann depuis le début des années 1990 avec des musiciens de jazz, et au premier chef Jean-Marc Padovani, au sein de la Grande Ritournelle. Sur ces jazz poems, voir Enzo Cormann. Le Mouvementeur, op. cit., p. 153-178 : «Jazz poem et écriture vocale ».

46. Enzo Cormann. L'Apothéose secrète, op. cit., p. 91.

47. Id., p. 7.

48. Cormann, Enzo. «La scène de crime des sœurs Papin. Notes sur la représentation-témoin du passage à l'acte catastrophique ». In: Le Geste de témoigner. Un dispositif pour le théâtre, textes réunis par Jean-Pierre Sarrazac, Catherine Naugrette et Georges Banu, Études théâtrales $\mathrm{n}^{\circ}$ 51-52, Louvain-la-Neuve, 2011, p. 144. Enzo Cormann s'est intéressé à l'affaire des sœurs Papin lors de l'écriture de sa pièce (inédite) Hors jeu en 2009-2010; il a également dirigé un atelier-recherche sur cette affaire à l'E.N.S.A.T.T. en mars 2011.

49. Cité par Enzo Cormann, id., p. 148.

50. Cormann, Enzo. «L'opération théâtrale », op. cit., p. 71. 


\section{RÉSUMÉS}

La question de l'entrée en scène est récurrente, voire insistante, dans l'œuvre d'Enzo Cormann : elle constitue un motif que son théâtre, sa pensée sur le théâtre mais également ses romans se plaisent à convoquer et à interroger, le plaçant dans des configurations contradictoires et le déployant au sein d'un vaste champ d'expériences aussi bien fictionnelles que théoriques. Il s'agit précisément, dans cet article, de mettre en lumière la complexité et l'ambivalence qui s'attachent à ce motif et à l'expérience qui en est faite. La modélisation qu'Enzo Cormann propose de l'entrée en scène est en lien avec sa pensée politique du théâtre : l'acteur, venu de l'assemblée théâtrale, s'en fait le délégué pour examiner, déplier, des questions collectives. Si ce projet prend corps dans la dramaturgie de plusieurs pièces, l'entrée en scène est pourtant souvent refusée par les personnages de son théâtre ou de ses romans, car elle y est associée à une expérience négative de la compromission. À travers les expériences multiples de l'entrée en scène ainsi déployées dans l'œuvre, ce qui ne cesse d'être interrogé, c'est la possibilité même du théâtre, sa vocation et son sens.

\section{INDEX}

Mots-clés : Cormann (Enzo), dramaturgie, poélitique, assemblée

\section{AUTEUR}

\section{MIREILLE LOSCO-LENA}

Professeur en études théâtrales à l'E.N.S.A.T.T., Lyon 